\title{
Uplink/Downlink Matching Based Resource Allocation for Full-Duplex OFDMA Wireless Cellular Networks
}

\author{
Tam Thanh Tran, Vu Nguyen Ha, Long Bao Le, and André Girard \\ INRS, University of Quebec, Montréal, Québec, Canada. \\ E-mails: \{tam.tran,hanguyen,long.le\}@emt.inrs.ca, andre.girard@gerad.ca.
}

\begin{abstract}
In this paper, we study the resource allocation problem for a full-duplex (FD) multiuser wireless system consisting of one FD base-station (BS) and multiple FD mobile nodes. Our main focus is to jointly optimize the power allocation (PA) and subcarrier assignment (SA) for both uplink (UL) and downlink (DL) transmissions of all users to maximize the system sum-rate. Our design captures the self-interference of FD transceivers and allows the utilization of each subcarrier for multiple concurrent UP and DL transmissions. Since the joint optimization problem is a nonconvex mixed integer program, which is difficult to tackle, we propose to employ the bipartite matching method to address the SA. Toward this end, a fast greedy allocation algorithm is developed to perform initial assignment of UL/DL links to each subcarrier that offers the best sum rate. Then from the obtained SA solution, we adopt the successive convex approximation approach to solve the PA problem whose results are used to calculate the SA weights for re-optimizing the SA by using the bipartite matching method. We then present the numerical results to demonstrate the improvement of our proposed algorithm in comparison with the greedy FD and half-duplex (HD) resource allocation algorithms.
\end{abstract}

Index Terms-OFDMA networks, full-duplex, resource allocation, sum rate maximization, bipartite matching

\section{INTRODUCTION}

Full-duplex (FD) communication has been regarded as a promising approach to significantly increase the capacity of next-generation wireless networks [1, 2]. In FD systems, data can be transmitted and received simultaneously on the same frequency band. Thus, FD systems can achieve twice the spectral efficiency of the half-duplex (HD) ones in ideal situation [1, 2]. The FD communications is made possible by the combination of various techniques, such as antenna design, analog cancellation, and digital cancellation, in order to effectively lower the self-interference (SI) between the transmitted and received signal on the same frequency band [3].

Nevertheless, resource allocation also has great influence on the performance of the FD systems [4] where there have been various studies on this topic. In [5], maximization of the minimum signal-to-interference-plus-noise (SINR) of all UL and DL links via power control is conducted. In [6], the user's weighted sum rate is maximized through the joint optimization of the time and power allocations. Nonetheless, these works only consider using the FD technique at the BS while the users are still in the HD mode and only the PA problem is examined.
Recently, a study on the joint optimization of PA and SA for the wireless network consisting FD base station and FD users is done in [7]. However, in this work, perfect SI cancellation is assumed, which is very difficult to achieve in a real system.

In this paper, we examine a single cell OFDMA network where all terminals (base station and users) have FD capability. Our objective is to maximize the system sum rate by jointly optimizing the SA and PA. Additionally, the SI in our paper is modeled as monomial form of transmission power instead of treating it as noise (eq. (8) in [8]). We propose to tackle the SA problem by using the bipartite matching method. Toward this end, an initial SA solution is determined by a fast greedy algorithm which allocates the maximum number of links to each subcarrier that offers the best sum rate with uniform power allocation. Then the successive convex approximation method (i.e., SCALE-based algorithm [9]) is applied to optimize the PA solution for the obtained SA solution based on which we calculate the SA weight matrix. Finally, we re-optimize the SA by solving the underlying matching problem and then employ the SCALE-based method for PA optimization in the final solution. Numerical studies are then conducted to demonstrate the desirable performance of the proposed matching based algorithm compared to other greedy FD and HD algorithms.

This paper is organized as follows. The FD system model and the sum-rate optimization problem are presented in Section II. The decoupled SA problem derived from our main problem and the algorithms to solve it are introduced in Section III. In Section IV, we look into the reformulated PA problem and the SCALE-based algorithm to find the PA solution. In Section V, we describe the algorithms based on the approaches presented in previous sections. Simulation results are presented in Section VI. Finally, we conclude this paper in Section VII.

\section{System Model AND Problem Formulation}

\section{A. System Model}

We consider a single cell OFDMA network consisting of one base station (BS) and $N$ mobile nodes all equipped with FD communication capability. Therefore, the BS and all the nodes are able to transmit and receive signals simultaneously on any subcarrier (SC). There are $S$ orthogonal SCs in the considered frequency band. Let $\mathcal{N}=\{1,2, \ldots, N\}$ and $\mathcal{S}=\{1,2, \ldots, S\}$ denote the set of nodes and the set of 
SCs, respectively. In this model, we also assume that each $\mathrm{SC}$ can be assigned to at most one user for UL transmission and also at most one user for DL transmission. The SA pattern for the UL and DL sides is defined by the binary vectors $\mathbf{X}^{\mathrm{U}} \triangleq\left\{x_{n, s}^{\mathrm{U}}\right\}_{n \in \mathcal{N}, s \in \mathcal{S}}$ and $\mathbf{X}^{\mathrm{D}} \triangleq\left\{x_{n, s}^{\mathrm{D}}\right\}_{n \in \mathcal{N}, s \in \mathcal{S}}$, respectively, where each component of $x_{n, s}^{\mathrm{U}}$ 's and $x_{n, s}^{\mathrm{U}}$ 's is defined as

$$
x_{n, s}^{\mathrm{U}}\left(\text { or } x_{n, s}^{\mathrm{D}}\right)=\left\{\begin{array}{l}
1 \text { if user } n \text { uses SC } s \text { for UL (or DL), } \\
0 \text { otherwise. }
\end{array}\right.
$$

Let $p_{n, s}^{\mathrm{U}}$ denote the UL power allocated to SC $s$ by node $n$ and $p_{n, s}^{\mathrm{D}}$ denote the DL power allocated to SC $s$ for node $n$ by BS. PA vector is defined as $\mathbf{P}$ as $\mathbf{P} \triangleq\left\{\mathbf{p}_{s}^{\mathrm{U}}, \mathbf{p}_{s}^{\mathrm{D}}\right\}_{s \in \mathcal{S}}$ where $\mathbf{p}_{s}^{\mathrm{U}}$ and $\mathbf{p}_{s}^{\mathrm{D}}$ are the uplink and downlink power vectors for SC $s$, respectively. The perfect channel information for each node and SC is assumed to be known by the BS. We can now start analyzing the rates achieved by all terminals in our systerm in order to define our problem.

1) Rate achieved at the base station: Because of the FD transmission, the received signal at BS over $\mathrm{SC} s$ is interfered by the DL transmitted signal from BS over that SC. Since the interference cancellation is not perfect, it won't be able to completely negate the interference. Thus, there exists residual self-interference in the SC. According to [8], the selfinterference at the BS from DL transmission corresponding to $\mathrm{SC} s$ can be formulated as

$$
I_{s}^{\mathrm{U}}\left(\mathbf{x}_{s}^{\mathrm{D}}, \mathbf{p}_{s}^{\mathrm{D}}\right)=\sum_{n \in \mathcal{N}} x_{n, s}^{\mathrm{D}} \epsilon\left(p_{n, s}^{\mathrm{D}}\right)^{\theta}
$$

where $\mathbf{x}_{s}^{\mathrm{D}}=\left\{x_{n, s}^{\mathrm{D}}\right\}_{n \in \mathcal{N}}$ is the DL SA vector for SC $s, \epsilon$ and $\theta$ are fixed scaling factors which captures the quality of the SI cancellation techniques. When $\theta=1$, the self-interference power is a linear function of the transmitted power [6]. When $\theta=0$, the self-interference is a constant regardless of the transmission power and thus can be treated as background noise [7]. Then, the rate achieved at the base station for the user $u$ on the UL transmission over SC $s$ can be computed as

$$
R_{n, s}^{\mathrm{U}}(\mathbf{X}, \mathbf{P})=\log \left(1+\frac{x_{n, s}^{\mathrm{U}} G_{n, s}^{\mathrm{U}} p_{n, s}^{\mathrm{U}}}{I_{s}^{\mathrm{U}}\left(\mathbf{x}_{s}^{\mathrm{D}}, \mathbf{p}_{s}^{\mathrm{D}}\right)+N_{0}}\right)
$$

where $G_{n, s}^{\mathrm{U}}$ is UL channel gain between node $n$ and the BS, and $N_{0}$ is the receiver noise power.

2) Rate achieved at the users: There are two cases which can happen when the user $n$ is assigned SC $s$ over DL transmission. In the first case, user $n$ also uses the same $\mathrm{SC} s$ for UL transmission. Therefore, this UL transmission creates self-interference and can be calculated as $\epsilon\left(p_{n, s}^{\mathrm{U}}\right)^{\theta}$. The second case is that SC $s$ is given to user $j$ for UL transmission. Then, in this case the UL transmission from user $j$ creates the cross-interference for the received DL signal of user $n$ over SC $s$, and this interference can be calculated as

$$
I_{j, n, s}^{\mathrm{cr}}\left(\mathbf{x}_{s}^{\mathrm{U}}, \mathbf{p}_{s}^{\mathrm{U}}\right)=x_{j, s}^{\mathrm{U}} G_{j, n, s} p_{j, s}^{\mathrm{U}},
$$

where $G_{n, j, s}$ is the interference channel gain from user $n$ to user $j$ over SC $s$ and $\mathbf{x}_{s}^{U}=\left\{x_{n, s}^{\mathrm{U}}\right\}_{n \in \mathcal{N}}$ is UL SA vector for
SC $s$. Generally, the interference on the received signal of user $n$ over SC $s$ can be summarized as follows.

$$
I_{n, s}^{\mathrm{D}}\left(\mathbf{x}_{s}^{\mathrm{U}}, \mathbf{p}_{s}^{\mathrm{U}}\right)=\sum_{j \in \mathcal{N} / n} x_{j, s}^{\mathrm{U}} G_{j, n, s} p_{j, s}^{\mathrm{U}}+x_{n, s}^{\mathrm{U}} \epsilon\left(p_{n, s}^{\mathrm{U}}\right)^{\theta} .
$$

Then, the rate achieved by the user $u$ on the DL transmission over SC $s$ can be computed as

$$
R_{n, s}^{\mathrm{D}}(\mathbf{X}, \mathbf{P})=\log \left(1+\frac{x_{n, s}^{\mathrm{D}} G_{n, s}^{\mathrm{D}} p_{n, s}^{\mathrm{D}}}{I_{n, s}^{\mathrm{D}}\left(\mathbf{x}_{s}^{\mathrm{U}}, \mathbf{p}_{s}^{\mathrm{U}}\right)+N_{0}}\right),
$$

\section{B. Problem Formulation}

In this section, we formulate the joint FD SA and PA problem based on the above analysis. This problem aims to calculate the SA for users in DL ans UL transmission as well as the corresponding power transmission for each link in order to maximize the total rate of the network. From these objectives, our problem can be formulated as

$$
\begin{aligned}
\max _{\mathbf{X}, \mathbf{P}} & R_{\text {total }}=\sum_{n \in \mathcal{N}} \sum_{s \in \mathcal{S}} R_{n, s}^{\mathrm{U}}(\mathbf{X}, \mathbf{P})+R_{n, s}^{\mathrm{D}}(\mathbf{X}, \mathbf{P}) \\
\text { s.t. } & \sum_{n \in \mathcal{N}} \sum_{s \in \mathcal{S}} p_{n, s}^{\mathrm{D}} \leq P_{\mathrm{BS}}, \\
& \sum_{s \in \mathcal{S}} p_{n, s}^{\mathrm{U}} \leq P_{n}, \forall n \in \mathcal{N}, \\
& \sum_{n \in \mathcal{N}} x_{n, s}^{\mathrm{U}} \leq 1 \text { and } \sum_{n \in \mathcal{N}} x_{n, s}^{\mathrm{D}} \leq 1, \forall s \in \mathcal{S}, \\
& x_{n, s}^{\mathrm{U}}, x_{n, s}^{\mathrm{D}} \in\{0,1\}, \forall n \in \mathcal{N}, \forall s \in \mathcal{S} .
\end{aligned}
$$

In this optimization problem, $P_{\mathrm{BS}}$ and $P_{n}$ denote the maximum transmission power budget that $\mathrm{BS}$ and user $n$ can use, respectively. The constraints (7d) impose the assignment of SC $s$ to at most one user for UL or DL transmissions. It can easily be seen that the optimization problem (7) is a mixed integer program, which is NP-hard in general and requires exponential complexity to be solved. Inspired by a recent work in PA and SA [9], we propose an iterative algorithm in the subsequent sections to find the locally optimal solution to problem (7). In the next section, we are going to introduce two SA methods, the fast greedy allocation with uniform power allocation and the bipartite matching method with the weights calculated using the result of the greedy algorithm.

\section{SA With Given PA OR UNiform POWER ALLOCATION}

\section{A. Greedy Allocation}

In this section, we introduce a fast greedy allocation algorithm for the SA problem. In this algorithm, each subcarrier will choose the group of nodes in both UL and DL transmission that offers the best rate, the constraint of maximum one UL and one DL transmission per subcarrier is ignored in this algorithm. Let $\mathcal{U}_{s}^{\mathrm{D}}$ and $\mathcal{U}_{s}^{\mathrm{U}}$ be the list of nodes assigned to subcarrier $s$ for DL and UL, respectively. The rate of subcarrier $s$ will be the sum of its users's rate. We will start analyzing the interference at each case of receiving terminal. 


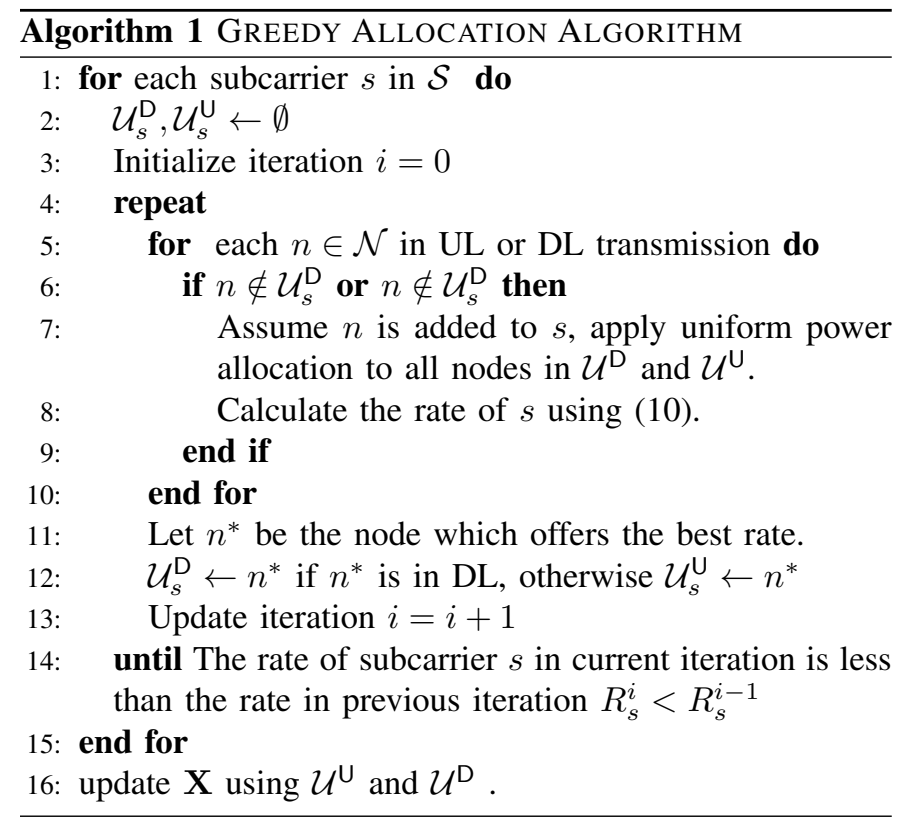

1) Interference at base station: At the base station, there are two sources of interference: from a node in UL mode or the SI from the base station transmitting in DL mode. Thus, the interference can be given as

$$
I_{j, s}^{\prime}(\mathbf{P})=\left\{\begin{array}{l}
\epsilon\left(p_{j, s}^{\mathrm{D}}\right)^{\theta}, j \in \mathcal{U}_{s}^{\mathrm{D}}, s \in \mathcal{S}, \\
G_{j, s}^{\mathrm{U}} p_{j, s}^{\mathrm{U}}, j \in \mathcal{U}_{s}^{\mathrm{U}}, s \in \mathcal{S} .
\end{array}\right.
$$

2) Interference at node: At the user, there are three sources of interference: from the base station broadcasting to another user in the same subcarrier, from another user in UL mode or the SI of that user in UL mode. Thus, the interference can be given as

$$
I_{j, n, s}^{\prime \mathrm{D}}(\mathbf{P})=\left\{\begin{array}{l}
G_{j, n, s} p_{j, s}^{\mathrm{U}}, j \in \mathcal{U}_{s}^{\mathrm{U}}, n \in \mathcal{U}_{s}^{\mathrm{D}}, s \in \mathcal{S}, j \neq n, \\
G_{j, s}^{\mathrm{D}} p_{j, s}^{\mathrm{D}}, j \in \mathcal{U}_{s}^{\mathrm{D}}, s \in \mathcal{S}, \\
\epsilon\left(p_{j, s}^{\mathrm{U}}\right)^{\theta}, j \in \mathcal{U}_{s}^{\mathrm{U}}, s \in \mathcal{S} .
\end{array}\right.
$$

3) Rate at subcarrier: From the above interference, the rate of a subcarrier $s$ can be calculated as follows:

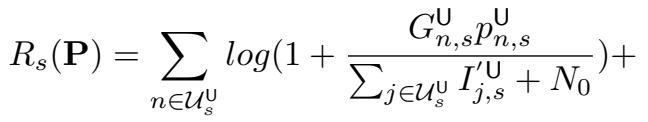

$$
\begin{aligned}
& \sum_{n \in \mathcal{U}_{s}^{\mathrm{D}}} \log \left(1+\frac{G_{n, s}^{\mathrm{D}} p_{n, s}^{\mathrm{D}}}{\sum_{j^{\prime} \in \mathcal{U}_{s}^{\mathrm{D}}} I_{j^{\prime}, n, s}^{\prime \mathrm{D}}+N_{0}}\right) .
\end{aligned}
$$

The algorithm uses uniform power allocation for all assigned nodes and subcarriers. The details of the greedy allocation are described in Algorithm 1.

\section{B. Bipartite Matching Formulation}

Since each subcarrier can have at most one DL transmission and UL transmission for $N$ nodes, there will be $N^{2}$ possible pairs of users in FD mode that can happen. As the subcarriers can also be used in HD mode, we will have another $2 N$ cases to consider. Thus, we define a $K \times K$ matrix $M$ where $K=$ $N^{2}+2 N$. The rows $\left\{1,2, . ., N^{2}\right\}$ in the matrix represent the pair of UL and DL nodes for the FD mode and rows $\left\{N^{2}+\right.$ $\left.1, \ldots, N^{2}+2 N\right\}$ are for the HD mode. The columns $\{1, \ldots, S\}$ represent the subcarriers and the rest of them are dummy in order for the matrix to be square. The value for each element of this matrix (each cell) is the rate obtained when the pair of nodes $(n, j)$ are assigned to subcarrier $s$.

For simplicity, new power variables $q_{s}^{\mathrm{U}}$ and $q_{s}^{\mathrm{D}}$ are introduced to represent the transmission power for all users on each SC for UL side and DL side, respectively. The relation between $q_{s}^{\mathrm{U}}$ and $p_{n, s}^{\mathrm{U}}, q_{s}^{\mathrm{D}}$ and $p_{n, s}^{\mathrm{D}}$ can be given as

$$
q_{s}^{\mathrm{U}}=\sum_{n \in \mathcal{N}} x_{n, s}^{\mathrm{U}} p_{n, s}^{\mathrm{U}}, \quad q_{s}^{\mathrm{D}}=\sum_{n \in \mathcal{N}} x_{n, s}^{\mathrm{D}} p_{n, s}^{\mathrm{D}} .
$$

Thus, the value of the cell on row $a^{t h}$, column $s^{t h}$ with $a \in$ $\left\{1, \ldots, N^{2}\right\}$ and $s \in \mathcal{S}$ can be calculated as

$$
\begin{aligned}
& W_{a, s}\left(q_{s}^{\mathrm{D}}, q_{s}^{\mathrm{U}}\right)=\log \left(1+\frac{q_{s}^{\mathrm{U}} G_{j, s}^{\mathrm{U}}}{\epsilon\left(q_{s}^{\mathrm{D}}\right)^{\theta}+N_{0}}\right) \\
& +\log \left(1+\frac{G_{n, s}^{\mathrm{D}} q_{s}^{\mathrm{D}}}{G_{j, n, s} q_{s}^{\mathrm{U}}+\epsilon_{s}^{\mathrm{U}}\left(q_{s}^{\mathrm{U}}\right)^{\theta}+N_{0}}\right),
\end{aligned}
$$

where $n=\lceil a / N\rceil$ and $j=a-\lfloor a / N\rfloor N$.

For rows $\left\{N^{2}+1, \ldots, N^{2}+N\right\}$ which are for DL transmission in HD mode, the values of the cell in columns $\{1, \ldots, S\}$ are

$$
W_{a, s}\left(q_{s}^{\mathrm{D}}\right)=+\log \left(1+\frac{G_{n, s}^{\mathrm{D}} q_{s}^{\mathrm{D}}}{N_{0}}\right),
$$

where $n=a-N^{2}$.

Rows $\left\{N^{2}+N+1, \ldots, N^{2}+2 N\right\}$ are the case of UL transmission in HD. The cell's values in columns $\{1, \ldots, S\}$ are

$$
W_{a, s}\left(q_{s}^{\mathrm{U}}\right)=+\log \left(1+\frac{G_{n, s}^{\mathrm{U}} q_{s}^{\mathrm{U}}}{N_{0}}\right),
$$

where $n=a-N^{2}$. The rest of matrix $M$ will be filled with 0. Fig. 1 shows the structure of our matching weight matrix.

Since $M$ is a square matrix with the values in each cell consider as "weight" for a subcarrier $s$ and we want to find the combination that offers the maximum rate, hence it is an assignment problem. Let $\tilde{x}_{a, s} \in \tilde{\mathbf{X}}$ be the binary assignment variable for our weight matrix $\mathcal{M}$, then the SA problem can be formulated as follows:

$$
\begin{aligned}
\max _{\tilde{\mathbf{X}}} & \sum_{a \in \mathcal{K}} \sum_{s \in \mathcal{S}} W_{a, s} \tilde{x}_{a, s} \\
& \sum_{a \in \mathcal{K}} \tilde{x}_{a, s}=1, \forall s \in \mathcal{S}, \\
& \tilde{x}_{a, s} \in\{0,1\}, \forall a \in \mathcal{K}, \forall s \in \mathcal{S} .
\end{aligned}
$$

This problem can be decomposed into multiple bipartite matching problems for individual subcarriers and UL/DL directions, which can be solved by the Kuhn-Munkres algorithm in polynomial time. 


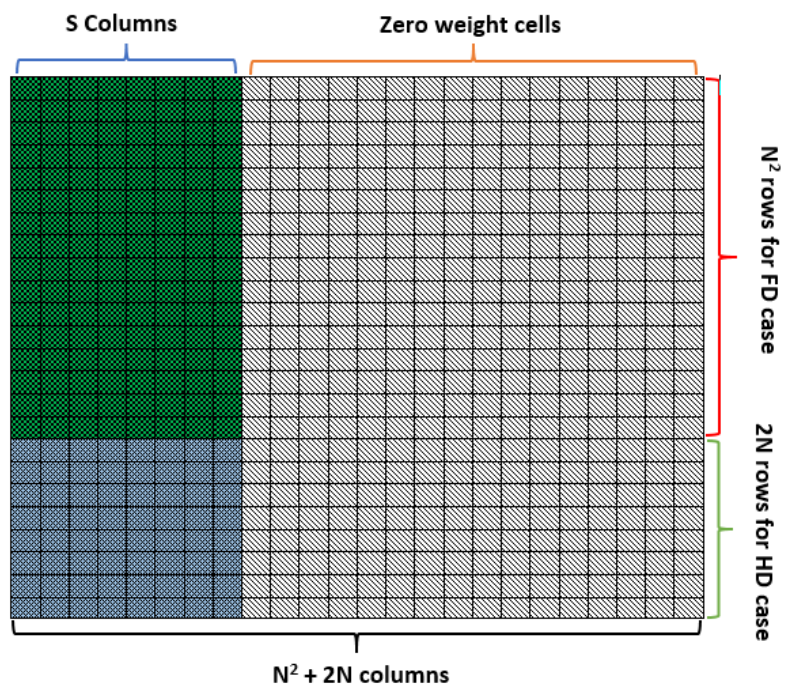

Fig. 1. Structure of the matching weight matrix.

\section{PA WITH GIVEN SA}

In this section, we study the PA problem and describe the SCALE-based algorithm in [9] to solve it. The SA solution from the greedy algorithm or the matching method will be used as input for this PA algorithm.

\section{A. Reformulation}

In order for the PA problem to be applied to a more general case when there are more than one source of interference, we will transform the objective function (7) by using (8) and (9). Thus, the interference becomes

$$
\begin{aligned}
I_{n, s}^{\prime \prime}(\mathbf{X}, \mathbf{P})= & \sum_{j \in \mathcal{N} / n} x_{j, s}^{\mathrm{U}} G_{j, s}^{\mathrm{U}} p_{j, s}^{\mathrm{U}}+\sum_{j^{\prime} \in \mathcal{N}} x_{j^{\prime}, s}^{\mathrm{D}} \epsilon\left(p_{j^{\prime}, s}^{\mathrm{D}}\right)^{\theta}, \\
I_{n, s}^{\prime \prime \mathrm{D}}(\mathbf{X}, \mathbf{P})= & \sum_{j \in \mathcal{N} / n}\left(x_{j, s}^{\mathrm{D}} G_{j, s}^{\mathrm{D}} p_{j, s}^{\mathrm{D}}+x_{j, s}^{\mathrm{U}} G_{j, n, s} p_{j, s}^{\mathrm{U}}\right) \\
& +x_{n, s}^{\mathrm{U}} \epsilon\left(p_{n, s}^{\mathrm{U}}\right)^{\theta} .
\end{aligned}
$$

The objective function with general interference is reformulated as

$$
\begin{aligned}
\max _{\mathbf{X}, \mathbf{P}} & R_{\mathrm{G}}=\sum_{n \in \mathcal{N}} \sum_{s \in \mathcal{S}} \log \left(1+\frac{x_{n, s}^{\mathrm{D}} G_{n, s}^{\mathrm{D}} p_{n, s}^{\mathrm{D}}}{I_{n, s}^{\prime \prime} \mathrm{D}(\mathbf{X}, \mathbf{P})+N_{0}}\right) \\
+ & \log \left(1+\frac{x_{n, s}^{\mathrm{U}} G_{n, s}^{\mathrm{U}} p_{n, s}^{\mathrm{U}}}{I_{n, s}^{\prime \prime} \mathrm{U}(\mathbf{X}, \mathbf{P})+N_{0}}\right) \\
\text { s.t. } & \sum_{n \in \mathcal{N}} \sum_{s \in \mathcal{S}} p_{n, s}^{\mathrm{D}} \leq P_{\mathrm{BS}}, \\
& \sum_{s \in \mathcal{S}} p_{n, s}^{\mathrm{U}} \leq P_{n}, \forall n \in \mathcal{N} .
\end{aligned}
$$

When the SA is given, this optimization problem is nonconvex which is difficult to calculate the optimal solution. Therefore, we will apply the SCALE method to solve it.

\section{B. SCALE-Based PA Algorithm}

From [9], we have the lower bound for any $z \geq 0$ and $\bar{z} \geq 0$ as

$$
\log (1+z) \geq \alpha_{\bar{z}} \log z+\beta_{\bar{z}},
$$

that is tight at $z=\bar{z}$, where

$$
\alpha_{\bar{z}}=\frac{\bar{z}}{1+\bar{z}}, \beta_{\bar{z}}=\log (1+\bar{z})-\frac{\bar{z}}{1+\bar{z}} \log \bar{z} .
$$

We use the convention that $\log (0)=-\infty$ and $0 \log (0)=0$.

Applying (23) to our objective function, we obtain

$$
\begin{aligned}
& R_{\mathrm{G}}(\mathbf{X}, \mathbf{P}) \geq \tilde{R}_{\mathrm{G}}(\mathbf{X}, \mathbf{P}, \alpha, \beta)= \\
& \sum_{n \in \mathcal{N}} \sum_{s \in \mathcal{S}}\left[\alpha_{n, s}^{\mathrm{D}} \log \left(\frac{x_{n, s}^{\mathrm{D}} G_{n, s}^{\mathrm{D}} p_{n, s}^{\mathrm{D}}}{I_{n, s}^{\prime \prime} \mathrm{D}(\mathbf{X}, \mathbf{P})+N_{0}}\right)+\beta_{n, s}^{\mathrm{D}}\right. \\
& \left.+\alpha_{n, s}^{\mathrm{U}} \log \left(\frac{x_{n, s}^{\mathrm{U}} G_{n, s}^{\mathrm{U}} p_{n, s}^{\mathrm{U}}}{I_{n, s}^{\prime \prime}(\mathbf{X}, \mathbf{P})+N_{0}}\right)+\beta_{n, s}^{\mathrm{U}}\right] .
\end{aligned}
$$

It can be noted that the bound is tight at

$$
\begin{aligned}
\bar{z}_{n, s}^{\mathrm{D}} & =\frac{x_{n, s}^{\mathrm{D}} G_{n, s}^{\mathrm{D}} p_{n, s}^{\mathrm{D}}}{I_{n, s}^{\prime \prime} \mathrm{D}(\mathbf{X}, \mathbf{P})+N_{0}}, \\
\bar{z}_{n, s}^{\mathrm{U}} & =\frac{x_{n, s}^{\mathrm{U}} G_{n, s}^{\mathrm{U}} p_{n, s}^{\mathrm{U}}}{I_{n, s}^{\prime \prime} \mathrm{U}(\mathbf{X}, \mathbf{P})+N_{0}} .
\end{aligned}
$$

By using transformation $\tilde{\mathbf{P}}=\log \mathbf{P}$, we have

$$
\begin{aligned}
& \tilde{R}_{\mathrm{G}}(\mathbf{X}, \tilde{\mathbf{P}}, \alpha, \beta)= \\
& \sum_{n \in \mathcal{N}} \sum_{s \in \mathcal{S}}\left[\alpha_{n, s}^{\mathrm{D}} \log \left(G_{n, s}^{\mathrm{D}}\right)+\beta_{n, s}^{\mathrm{D}}+\alpha_{n, s}^{\mathrm{D}} \tilde{p}_{n, s}^{\mathrm{D}}\right. \\
& -\alpha_{n, s}^{\mathrm{D}} \log \left(\sum_{j \in \mathcal{N} / n}\left(x_{j, s}^{\mathrm{D}} G_{j, s}^{\mathrm{D}} e^{\tilde{p}_{n, s}^{\mathrm{D}}}+x_{j, s}^{\mathrm{U}} G_{j, n, s} e^{\tilde{p}_{n, s}^{U}}\right)\right. \\
& \left.+x_{n, s}^{\mathrm{U}} \epsilon e^{\tilde{p}_{n, s}^{U} \theta}+N_{0}\right)+\alpha_{n, s}^{\mathrm{U}} \log \left(G_{n, s}^{\mathrm{U}}\right)+\beta_{n, s}^{\mathrm{U}} \\
& +\alpha_{n, s}^{\mathrm{U}} \tilde{p}_{n, s}^{\mathrm{U}}-\alpha_{n, s}^{\mathrm{U}} \log \left(\sum_{j \in \mathcal{N} / n} x_{j, s}^{\mathrm{U}} G_{j, s}^{\mathrm{U}} e^{\tilde{p}_{j, s}^{U}}\right. \\
& \left.\left.+\sum_{j^{\prime} \in \mathcal{N}} x_{j^{\prime}, s}^{\mathrm{D}} \epsilon e^{\tilde{p}_{j^{\prime}, s}^{\mathrm{D}} \theta}+N_{0}\right)\right] .
\end{aligned}
$$

It can be verified that $\tilde{R_{\mathrm{G}}}(\mathbf{X}, \mathbf{P}, \alpha, \beta)$ is a concave function of $\tilde{\mathbf{P}}$. Then, for given $\alpha, \beta$ and $\mathbf{X}$, the problem (20) can be described as follows:

$$
\begin{aligned}
\max _{\tilde{\mathbf{P}}} & \tilde{R}_{\mathrm{G}}(\mathbf{X}, \mathbf{P}, \alpha, \beta) \\
\text { s.t. } & \sum_{s \in \mathcal{S}} e^{\tilde{p}_{n, s}^{\mathrm{D}} \leq P_{\mathrm{BS}},} \\
& \sum_{s \in \mathcal{S}} x_{n, s}^{U} e^{\tilde{p}_{s}^{U}} \leq P_{n}, \forall n \in \mathcal{N} .
\end{aligned}
$$

The problem is a standard convex maximization problem that can be solved optimally by any standard methods. After we have found the optimal value of $\tilde{\mathbf{P}}, \mathbf{P}$ can obtained by using $\mathbf{P}=\exp (\tilde{\mathbf{P}})$. The algorithm aims to optimize the lower bound of $\tilde{R}_{\mathrm{G}}(\mathbf{X}, \tilde{\mathbf{P}}, \alpha, \beta)$. Therefore, we need to tighten the bound successively by using the new power transmission solution to 

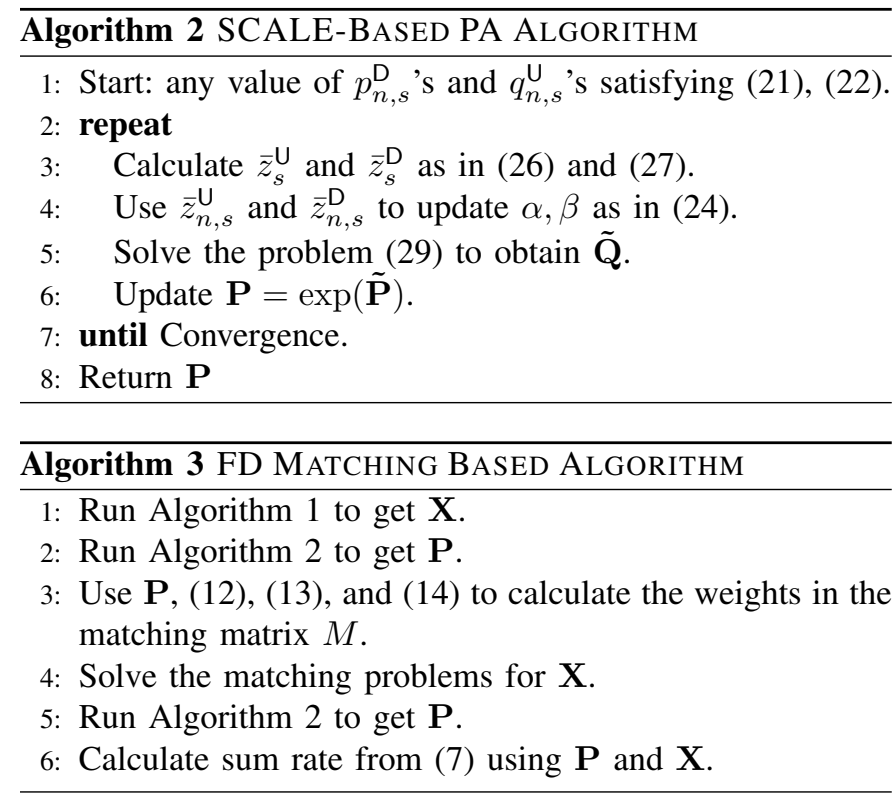

update the choice of $\alpha, \beta$. The process of iteratively updating $\bar{z}_{s}^{\mathrm{U}}$ 's, $\bar{z}_{s}^{\mathrm{D}}$ 's as well as $\alpha, \beta$, is the SCALE-based PA algorithm to maximize DL and UL sum-rate, which is summarized in Algorithm 2. The the algorithm is guaranteed to converge as shown in the following Proposition.

Proposition 1. The Algorithm 2 converges after a finite number of iterations.

Proof. The process of iteratively updating $\bar{z}_{s}^{\mathrm{U}}$ 's, $\bar{z}_{s}^{\mathrm{D}}$ 's, and $\alpha, \beta$ as in (26), (27), and (24) will always guarantee that the optimal solution of problem (29) in the previous iteration is a feasible point in the feasible set of that problem in the next iteration. Thus, the algorithm is guaranteed to converge due to the monotonic increase of the objective function in problem (29) after each iteration. Hence, Algorithm 2 converges after a finite number of iterations.

\section{Resource Allocation Algorithms}

In this section, we will present the algorithms that will be used in the simulation.

\section{A. Proposed FD Matching Based Algorithm}

In our proposed FD matching based algorithm, the SA problem is solved by using the matching method and the PA problem will be solved by the SCALE-based algorithm. In order to compute the weights for the matching matrix, a fast greedy allocation will be employed to find an initial SA solution and then the initial PA solution for calculating weights can be found by applying the SCALE method. The detail of the proposed algorithm is shown in Algorithm 3.

\section{B. FD Greedy Algorithm}

This simple algorithm is considered as the input step for finding the initial weights in our proposed FD matching based algorithm. Algorithm 1 is used to find the SA solution, then we compute the PA solution by using Algorithm 2.

\section{HD Greedy Algorithm}

In this algorithm, each SC is assigned at most one link, either UL or DL transmission. This algorithm is similar to Algorithm 1 where the uniform power distribution is employed for this method as well. For SC allocation, each SC is assigned for the user with its DL or UL transmission corresponding the highest achievable rate instead of a pair of UL and DL transmissions as in the FD algorithm.

\section{Simulation Results}

We consider a single cell network in which the users are placed randomly. The channel gains are generated by considering both Rayleigh fading and path loss which is modeled based on the WINNER-II Model as $P L(d)=A \log _{10}(d)+B+$ $C \log _{10}\left(f_{c} / 5\right)$, where $d$ is the distance between two terminals. The detail of the parameters used in our simulation is shown in Table I. In Figs. 3, the maximum powers of the BS and nodes will be multiplied with a scaling factor $\Omega$ to analyze the effect of power on the system's sum rate.

TABLE I

Simulation System PARAMETERS

\begin{tabular}{|l|l|}
\hline \hline Parameters & Value \\
\hline \hline WINNER-II Model's parameters & $\begin{array}{l}\mathrm{A}=36.8, \mathrm{~B}=43.8, \mathrm{C}=23, \\
\text { and } f_{c}=2.5 \mathrm{GHz}\end{array}$ \\
\hline Minimum distance & $50 \mathrm{~m}$ \\
\hline Maximum distance & $100 \mathrm{~m}$ \\
\hline Number of nodes & 10 \\
\hline Number of carriers & 20 \\
\hline Power of base station & $4 \mathrm{~W}$ \\
\hline Power of node & $0.4 \mathrm{~W}$ \\
\hline Noise power $N_{0}$ & $10^{-} 12 \mathrm{~W}$ \\
\hline$\epsilon$ & $10^{-} 12$ \\
\hline$\theta$ & 0.5 \\
\hline \hline
\end{tabular}

In Figs. 2-5, we show the variations of the sum rate achieved by our proposed algorithm and two greedy algorithms presented in the previous section - "FD Greedy Alg." and "HD Greedy Alg." over the changes of some network parameters, e.g., the interference factor $\epsilon$ in Fig. 2, the maximum transmission power in Fig. 3, the number of SCs in Fig. 4, and the number of nodes in Fig. 5. As it can been seen from these figures, the proposed FD matching based algorithm surpasses the two greedy algorithms in all studied scenarios. Additionally, we can also see that both of the FD algorithms achieved much better sum rate than the HD one.

As can be seen in Fig. 3, the higher value of interference factor $\epsilon$ results in the lower sum rate of our system when it is in FD mode ("Proposed Alg." and "FD Greedy Alg."). This can be easily explained since the higher value of $\epsilon$ means that the SI cancellation is less effective in preventing the interference. Thus it results in lowering the advantages of FD technique. In Fig. 3, increasing the transmission powers of BS and nodes via increasing scaling factor $\Omega$ also yields a higher sum rate achieved by the network as expected.

Fig. 4 and Fig. 5 also show that the higher number of SCs or higher number of nodes also lead to the higher network 


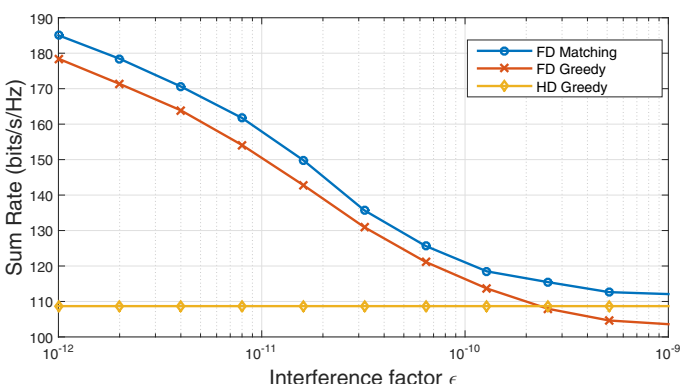

Fig. 2. Total rate versus the interference factor $\epsilon$.

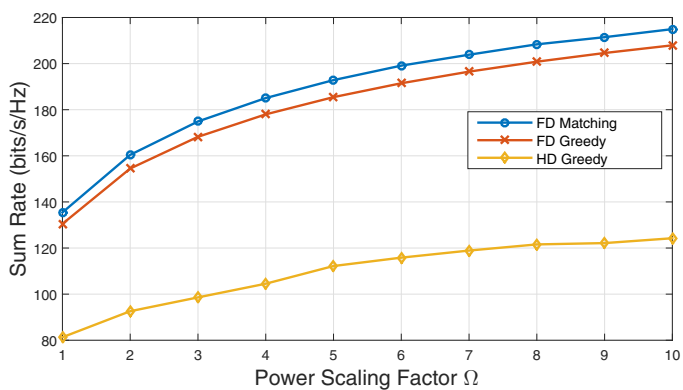

Fig. 3. Sum rate versus the power scaling factor $\Omega$.

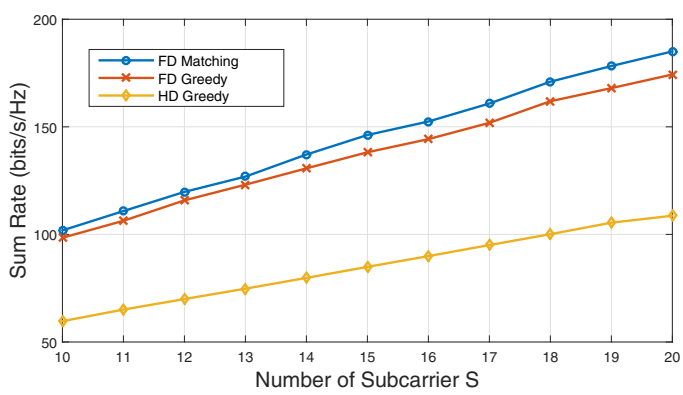

Fig. 4. Sum rate versus the number of subcarriers.

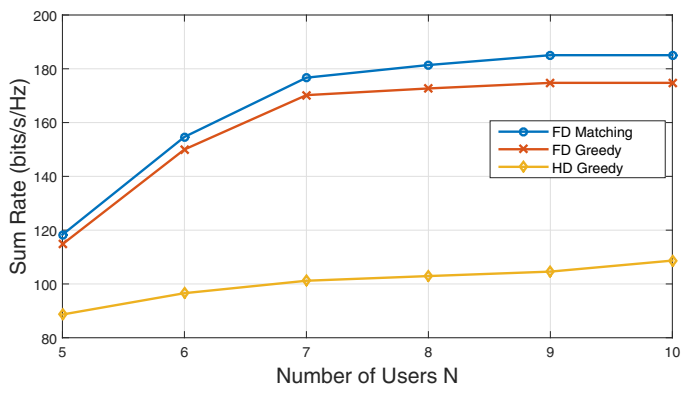

Fig. 5. Sum rate versus the number of users.

sum rate. The results from Fig. 2 to Fig. 5 also show that the sum rate due to the proposed FD matching based algorithm is about $5 \%$ higher than that of the FD greedy algorithm and almost twice the sum rate of the HD greedy algorithm.

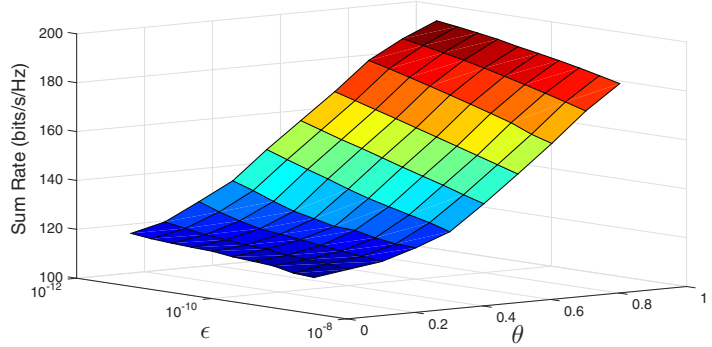

Fig. 6. Sum rate versus epsilon versus theta.

Fig. 6 demonstrates the influences of the SI cancellation parameters $\epsilon$ and $\theta$ on the sum rate of our proposed algorithm. It can be seen that the smaller value of $\theta$ results in the lower sum rate. This is because the power value is less than 1 , so when a value of $0 \leq \theta \leq 1, p^{\theta}$ is bigger than the transmission power $p$.

\section{CONCLUSION}

In this paper, we have proposed an FD matching based algorithm for joint PA and SA design in FD single cell network with the goal to maximize the system's sum rate subject to constraints on the power transmission limit. The SA is solved using the bipartite matching method and the PA's solution is found by the SCALE-based algorithm. Numerical results have illustrated the efficiency of our proposed algorithms and the influences of different parameters on the performance of our algorithm.

\section{REFERENCES}

[1] D. Kim, H. Lee, and D. Hong, "A survey of in-band full-duplex transmission: From the perspective of PHY and MAC layers," IEEE Commun. Surveys Tuts., vol. 17, no. 4, pp. 2017-2046, 4th Quarter 2015.

[2] M. Duarte and A. Sabharwal,"Full-duplex wireless communications using off-the-shelf radios: Feasibility and first results," in Proc. of IEEE ASILOMAR, 2010.

[3] E. Everett, A. Sahai, and A. Sabharwal, "Passive self-interference suppression for full-duplex infrastructure nodes," IEEE Trans. Wireless Commun., vol. 13, no. 2, pp. 680-694, Feb. 2014.

[4] L. Song, Y. Li, and Z. Han, "Resource allocation in full-duplex communications for future wireless networks," IEEE Wireless Commun., vol. 22, no. 4, pp. 88-96, Aug. 2015.

[5] W. Choi, H. Lim, and A. Sabharwal, "Power-controlled medium access control protocol for full-duplex wifi networks," IEEE Trans. Wireless Commun., vol. 14, no. 7, pp. 3601-3613, May 2015.

[6] H. Ju and R. Zhang, "Optimal resource allocation in full-duplex wirelesspowered communication network," IEEE Trans. Commun., vol. 62, no. 10, pp. 3528-3540, Oct. 2014.

[7] C. Nam, C. Joo and S. Bahk, "Joint subcarrier assignment and power allocation in full-duplex OFDMA networks," IEEE Trans. Wireless Commun., vol. 14, no. 6, pp. 3108-3119, Jun. 2015.

[8] L. Jiménez Rodríguez, N. H. Tran, and T. Le-Ngoc, "Performance of full-duplex AF relaying in the presence of residual self-interference," IEEE J. Select. Areas in Commun., vol. 32, no. 9, pp. 1752-1764, Sept. 2014.

[9] L. Venturino, N. Prasad, and X. Wang, "Coordinated scheduling and power allocation in downlink multicell OFDMA networks," IEEE Trans. Veh. Technol., vol. 58, no. 6, pp. 2835-2848, Jul. 2009.

[10] M. Sanjabi, M. Razaviyayn, and Zhi-Quan Luo, "A unified convergence analysis of block successive minimization methods for nonsmooth optimization," SIAM J. Opt., , vol. 23, no. 2, pp. 1126-1153, 2013. 